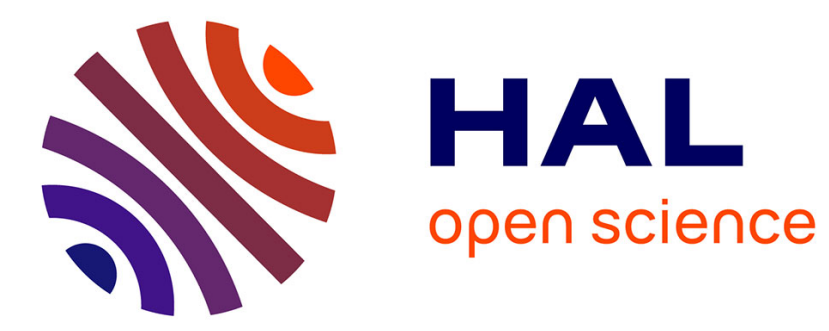

\title{
Le Carici flaccae-Quercetum roboris ass. nov. Une nouvelle association phytosociologique en région Centre
}

Alain Brêthes

\section{To cite this version:}

Alain Brêthes. Le Carici flaccae-Quercetum roboris ass. nov. Une nouvelle association phytosociologique en région Centre. Revue forestière française, 2010, 62 (3-4), pp.247-260. $10.4267 / 2042 / 38939$. hal-03449618

\section{HAL Id: hal-03449618 https://hal.science/hal-03449618}

Submitted on 25 Nov 2021

HAL is a multi-disciplinary open access archive for the deposit and dissemination of scientific research documents, whether they are published or not. The documents may come from teaching and research institutions in France or abroad, or from public or private research centers.
L'archive ouverte pluridisciplinaire HAL, est destinée au dépôt et à la diffusion de documents scientifiques de niveau recherche, publiés ou non, émanant des établissements d'enseignement et de recherche français ou étrangers, des laboratoires publics ou privés. 


\title{
Le CaRici flaccae-QUeRCETUM Roboris ass. NOV. UNE NOUVELLE ASSOCIATION PHYTOSOCIOLOGIQUE EN RÉGION CENTRE
}

\author{
Alain Brêthes
}

Si l'association du Molinio caeruleae-Quercetum roboris (Tüxen, 37) Scamoni et Passarge 59 est bien connue et décrite dans de nombreuses régions, il existe, en région Centre, des stations où se trouvent en mélange, en particulier, la Molinie bleue (Molinia caerulea (L.) Moench), le Brachypode penné (Brachypodium pinnatum (L.) Beauv.) et la Laîche glauque (Carex flacca Schreb.). Cette combinaison d'espèces est impossible dans le contexte de l'association précédente.

Aucune association phytosociologique actuellement décrite ne correspond à cet ensemble relativement original (Rameau, communication personnelle). Nous proposons, de ce fait, la création d'une nouvelle association le Carici flaccae-Quercetum roboris ass. nov.

Mais le rattachement de cette association aux diverses alliances retenues dans le prodrome des végétations de France (Bardat et al., 2004) présente quelques difficultés qu'il faut éclaircir.

\section{CARACTÈRES FLORISTIQUES : ASSOCIATION DE LA MOLINIE BLEUE, DE LA LAÎCHE GLAUQUE ET DU BRACHYPODE PENNÉ}

Au cours des études des stations forestières dans l'Orléanais (Brêthes, 1993) et surtout en Champagne berrichonne et Boischaut-sud (Brêthes, 2003), nous avons observé un groupement végétal original comportant (tableau III, pp. 256-260) à la fois des espèces considérées comme calcicoles, neutro-calcicoles ou neutroclines: Prunus spinosa, Rosa canina, Rosa arvensis, Carex flacca, Brachypodium pinnatum, Ligustrum vulgare, Serratula tinctoria, Cornus sanguinea, Crataegus laevigata, etc. ainsi que, sur les sols les plus carbonatés, Lonicera xylosteum, Viburnum lantana, Berberis vulgaris, Juniperus communis, etc. et d'autres appartenant aux cortèges des espèces acidiphiles et hygro-acidiphiles : Calluna vulgaris, Deschampsia flexuosa, Melampyrum pratense, Pteridium aquilinum, Teucrium scorodonia, Frangula alnus, Peucedanum gallicum, Asphodelus albus, etc. et surtout Molinia caerulea.

C'est bien l'association de ces deux ensembles d'espèces qui constitue l'originalité du groupement.

Trois espèces sont considérées comme caractéristiques de cette nouvelle association : la Molinie bleue, le Brachypode penné et la Laîche glauque. Les deux premières espèces semblaient les plus logiques pour l'appellation de cette association. Mais tant le nom de Molinio caeruleaeQuercetum roboris que celui de Brachypodio pinnati-Quercetum roboris ont déjà été utilisés, chênaie pédonculée humide à Molinie dans le premier cas (Scamoni et Passarge 59) et chênaie xérophile du sud de l'Oural relevant du Lathyro-Quercion (Solomeshch et al., 1989) dans le second. Le nom proposé repose donc sur la troisième espèce caractéristique Carex flacca. Nous proposons donc le nom de Carici flaccae-Quercetum roboris ass. nov. 
ALAIN BRÊTHES

\section{UN CONTEXTE STATIONNEL PARTICULIER}

\section{Sa géologie et ses sols}

Ce groupement végétal se rencontre en position de replat en bas de versant ou dans des cuvettes sur deux groupes de matériaux. D'une part des formations très argileuses dès la surface du sol, ce sont les situations les plus caractéristiques de cette association (17 premiers relevés dans le tableau III), et, d'autre part, des formations limoneuses, limono-sableuses ou sableuses en surface mais reposant sur des argiles à faible profondeur (en général moins de $25 \mathrm{~cm}$ d'épaisseur). Les matériaux argileux ont, en moyenne, une très bonne richesse minérale et sont parfois carbonatés en profondeur.

En région Centre, trois formations géologiques sont à l'origine de ces sols : les argiles de l'Orléanais (Burdigalien-Miocène) pour la forêt d'Orléans, les argiles de Lignières (Eocène) et les marnes lacustres du Berry (Oligocène) pour les forêts du Boischaut-sud.

La nature géologique des matériaux et la situation topographique de ces stations entraînent un régime hydrique très contrasté d'une saison à l'autre. Ces sols sont gorgés d'eau, présence parfois de mares en surface des sols, lors des périodes pluvieuses, et extrêmement secs, avec de nombreuses fentes de retrait dans les matériaux argileux, en période estivale. L'engorgement est donc bien un engorgement primaire et non pas résultant d'une dégradation des sols. Ils se rattachent, pour les formations les plus argileuses, aux Brunisols-Rédoxisols parfois vertiques ou aux Pélosols-Rédoxisols et, pour les formations à texture plus grossière en surface, aux Planosols. Les humus sont de forme eumull, mésomull ou hydromull.

\section{Ses peuplements}

Sur ces stations, les peuplements de Chêne ont une croissance limitée. La strate arborescente est constituée essentiellement par les Chênes pédonculé et sessile. Parfois accompagnés par l'Alisier torminal, l'Érable champêtre, les Bouleaux et le Tremble. Ces peuplements sont rarement bien venants. Les arbres sont courts et de faible diamètre. Quelques plantations de Pin sylvestre ont été observées. Elles sont souvent de belle venue.

Le taillis est constitué, outre les essences précédentes, de Bourdaine et de nombreux ligneux calcicoles et neutro-calcicoles : Prunellier, Aubépines monogyne et épineuse, Cornouiller sanguin, Rosiers, Troène, ainsi que, parfois, Genévrier, Camérisier à balais, Viorne lantane, Épine-vinette, Spirée à feuilles de millepertuis.

\section{Son climat}

Le climat de cette région ligérienne se caractérise surtout par ses étés secs.

Tant dans l'Orléanais que dans le Sud Berry, la pluviométrie est relativement réduite (moins de $750 \mathrm{~mm}$ annuel) avec un déficit de pluviométrie marqué en période estivale (moins de $200 \mathrm{~mm}$ pour la période juin, juillet, août). L’humidité atmosphérique estivale est faible ( 50 \% en général).

Les températures moyennes annuelles sont douces (10 à $11{ }^{\circ} \mathrm{C}$ ) avec des étés relativement chauds (moyenne de 18 à $20^{\circ} \mathrm{C}$ en juillet et août mais les maximales peuvent atteindre ou dépasser $25^{\circ} \mathrm{C}$ au mois de juillet ou d'août) et des hivers doux (moyenne de 3 à $4^{\circ} \mathrm{C}$ en janvier avec des valeurs minimales moyennes rarement inférieures à $0^{\circ} \mathrm{C}$ ).

Ceci conduit à des valeurs de l'ETP largement supérieures à la pluviométrie dès le mois de juin et jusqu'au mois de septembre. Le déficit hydrique cumulé en fin d'été, estimé par la formule de Thornthwaite, se situe en moyenne entre 170 et $200 \mathrm{~mm}$. 


\section{LES LIENS AVEC D'AUTRES ASSOCIATIONS FORESTIÈRES ET PROPOSITION DE RATTACHEMENT}

Plusieurs associations végétales proches de l'association du Carici flaccae-Quercetum roboris ass. nov. ont été reconnues dans la région Centre (Gauberville, 2003). Il s'agit de la chênaie sessiliflore ligérienne à Alisier torminal (Sorbo torminalis-Quercetum petraeae Géhu et al. 74), de la chênaie pédonculée acidiphile hydromorphe à Molinie (Molinio caeruleae-Quercetum roboris (Tüxen, 37) Scamoni et Passarge 59), de la chênaie pédonculée à Peucédan de France (Peucedano gallici-Quercetum roboris Br Bl. $67 \mathrm{pp}$ ) toutes rattachées aux ordres du Quercion, mais aussi la chênaie pédonculée ligérienne à Fragon petit houx (Rusco-Quercetum petraeae (Noirfalise 69 pp) Rameau 96) rattaché à l'ordre du Carpinion betuli (Issler 31).

Une analyse factorielle des correspondance (AFC) réalisée sur les relevés types de huit associations régionales caractéristiques (figure 1, ci-dessous) montre les proximités existant entre l'association du Molinio caeruleae-Quercetum roboris (Tüxen, 37) Scamoni et Passarge 59 et celle du Peucedano gallici-Quercetum roboris selon Braun-Blanquet (1967) et le Carici flaccae-Quercetum roboris ass. nov. et, surtout, la conservation de ces proximités sur les différents plans factoriels étudiés. Au contraire, on note une certaine dispersion pour les autres associations. Ceci tient très certainement à la présence de la Molinie bleue et des espèces à caractère ligérien.

L'association du Molinio caeruleae-Quercetum roboris (Tüxen, 37) Scamoni et Passarge 59, appartenant à l'alliance du Molinio-Quercion, comporte les mêmes espèces acidiphiles mais très rarement les espèces calcicoles et neutrocalcicoles. Ce caractère nettement acidiphile du groupement et l'hydromorphie prononcée de ses sols ont été soulignés par de nombreux auteurs. Cette association se différencie également par la nature de ses sols. En effet, elle correspond à des sols à alternance hydrique marquée mais particulièrement acides. En région Centre, il s'agit souvent de sols

FIGURE 1
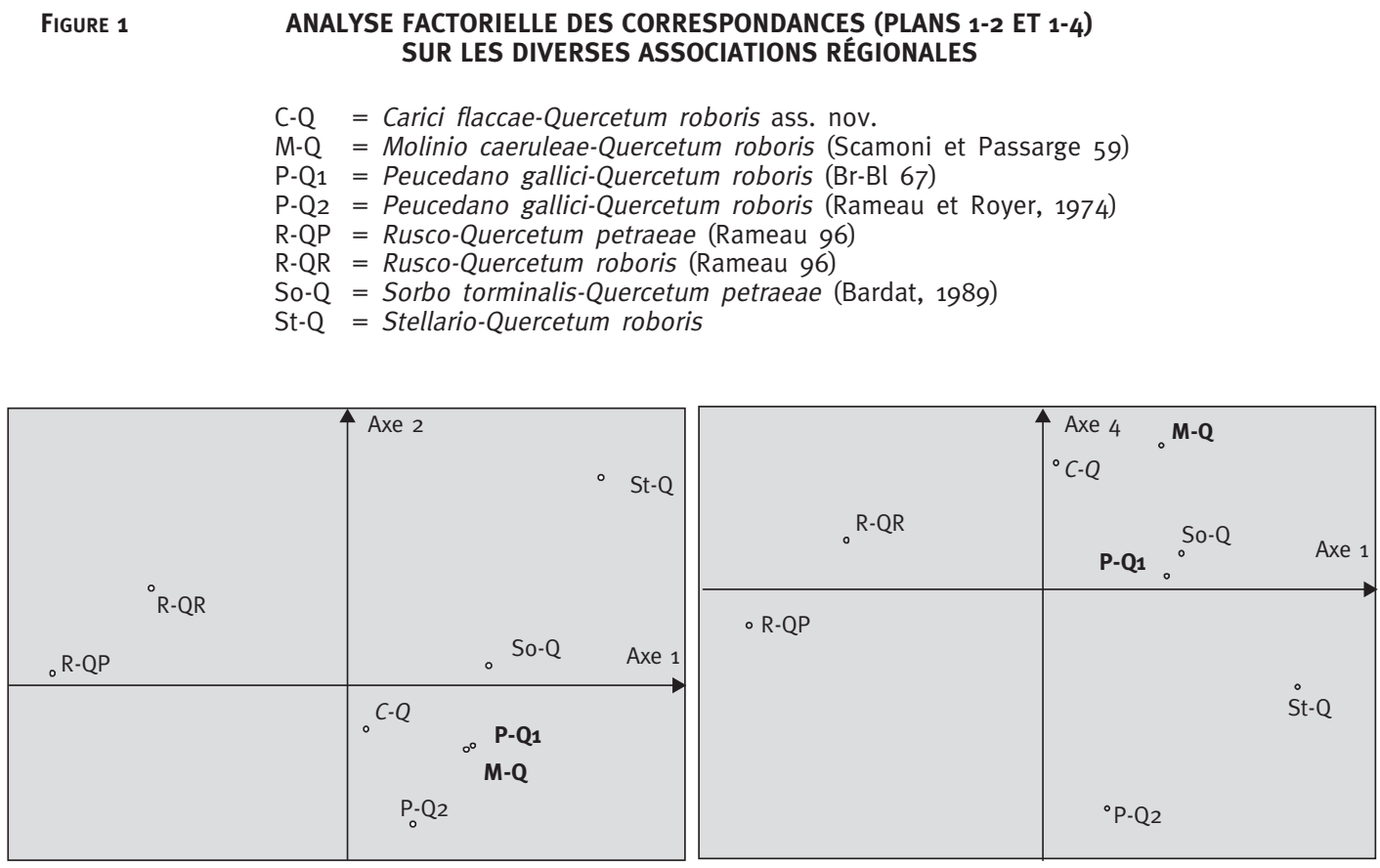
développés sur des sables plus ou moins épais (forêts d'Orléans, de Sologne et du Berry) ou sur des limons acides (forêts du Berry) à humus de forme moder, hydromoder, mor ou hydromor.

Le rapprochement avec l'association du Rusco aculeati-Quercetum roboris (Charnet 88) Rameau 96 repose sur la situation topographique favorisant les excès d'eau et la richesse minérale des sols. Mais notre association s'en distingue, entre autres, par le régime hydrique contrasté des sols et par la présence de la Molinie bleue et la rareté du Charme.

Le rapprochement avec le Peucedano gallici-Quercetum roboris selon Braun-Blanquet (1967) ou selon Rameau et Royer (1974) est tout à fait possible en raison de la présence régulière du Peucédan de France et de l'Alisier torminal. Toutefois cette association ne comporte pas d'espèces neutro-calcicoles.

Celui avec le Sorbo torminalis-Quercetum petraeae (Géhu et al. 74) Bardat em. Rameau 97 n'est guère envisageable. En effet, cette association ne concerne pas les groupements développés sur des sols à engorgement prononcé et ne possède ni Molinie bleue ni Brachypode penné et Laîche glauque.

Les rattachements à l'ordre des Fagetalia sylvaticae ou à l'alliance du Fraxino excelsioris-Quercion roboris ont été rejetés en raison soit de l'absence d'espèces caractéristiques soit d'un régime hydrique particulier.

Le tableau I (pp. 251-252) permet une comparaison des fréquences des espèces caractéristiques de ces diverses associations. On y remarque que l'association du Carici flaccae-Quercetum roboris ass. nov. s'individualise nettement des autres associations justement par la fréquence régulièrement importante des deux groupes d'espèces : Molinie + Bourdaine et Brachypode penné + Laîche glauque et Prunellier.

Le rattachement de cette nouvelle association à l'alliance du Molinio-Quercion nécessiterait de modifier assez nettement la définition de cette alliance en créant deux pôles : l'un en milieu

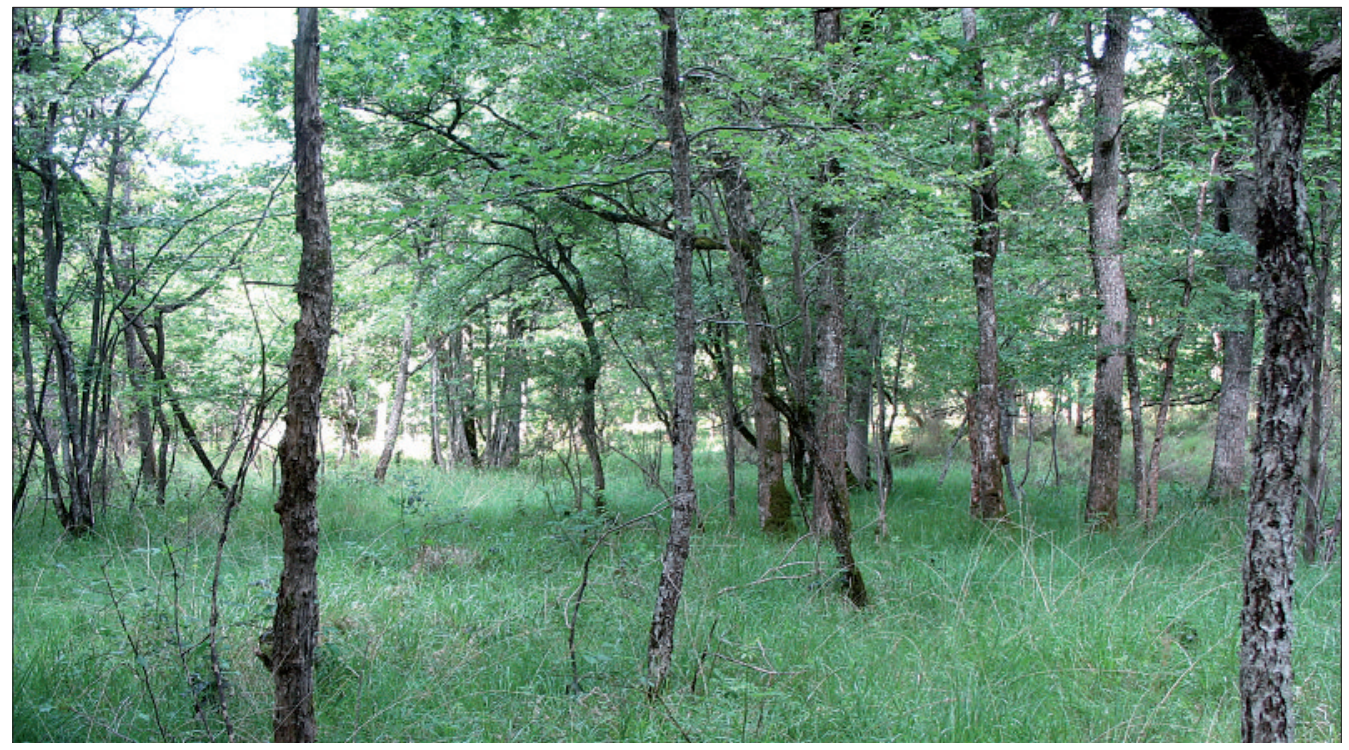

Photo 1 Carici flaccae-Quercetum roboris en forêt de Chœurs-Bommiers (Indre), parcelle 74. 
Fréquences des espèces végétales dans les diverses associations phytosociologiques proches

\begin{tabular}{|c|c|c|c|c|c|c|c|}
\hline & 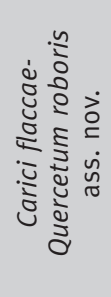 & 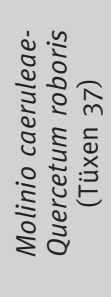 & 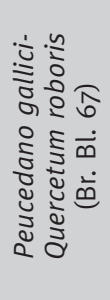 & 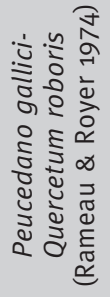 & 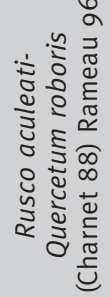 & 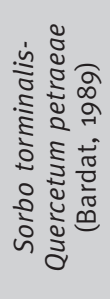 & 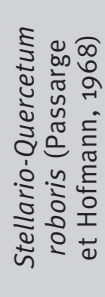 \\
\hline Quercus petraea .... & III & III & 1 & V & $\mathrm{I}$ & $\mathrm{V}$ & II \\
\hline Quercus robur...... & $\mathrm{V}$ & III & V & IV & IV & $\mathrm{I}$ & $\mathrm{V}$ \\
\hline Carpinus betulus .... & II & 1 & & IV & $\mathrm{V}$ & II & II \\
\hline Betula pendula ..... & 1 & III & III & IV & II & 1 & IV \\
\hline Betula pubescens ... & & II & & III & & III & IV \\
\hline Fagus sylvatica . . . . . & & 1 & & II & & IV & V \\
\hline Populus tremula .... & III & II & II & & II & 1 & \\
\hline Rubus fruticosus .... & $\mathrm{V}$ & $\mathrm{V}$ & & $\mathrm{V}$ & IV & $\mathrm{V}$ & $\mathrm{V}$ \\
\hline Frangula alnus ..... & $\mathrm{V}$ & $\mathrm{V}$ & II & $\mathrm{V}$ & & II & $\mathrm{V}$ \\
\hline $\begin{array}{l}\text { Lonicera } \\
\text { periclymenum . . . . . }\end{array}$ & $\mathrm{V}$ & IV & $\mathrm{V}$ & $\mathrm{V}$ & III & $\mathrm{V}$ & IV \\
\hline Corylus avellana .... & II & 1 & II & III & IV & III & II \\
\hline Deschampsia flexuosa & II & III & III & V & + & V & IV \\
\hline Pteridium aquilinum. . & $\mathrm{I}$ & $\mathrm{I}$ & $\mathrm{I}$ & III & + & IV & $\mathrm{V}$ \\
\hline $\begin{array}{l}\text { Brachypodium } \\
\text { pinnatum ........ }\end{array}$ & V & & & & & & \\
\hline Carex flacca. . . . . . . & IV & + & & & & & \\
\hline Prunus spinosa . . . . . & V & + & & & I & & \\
\hline Pulmonaria longifolia & IV & + & & & II & & \\
\hline Rosa canina ...... & II & I & & & & & \\
\hline Viburnum opulus.... & III & 1 & & & + & & \\
\hline Rosa arvensis ..... & IV & I & & & III & + & \\
\hline Asphodelus albus ... & II & 1 & & & & & \\
\hline $\begin{array}{l}\text { Rhytidiadelphus } \\
\text { triquetrus . . . . . . }\end{array}$ & 1 & I & I & & & + & \\
\hline Peucedanum gallicum & IV & II & 1 & IV & & & \\
\hline Molinia caerulea .... & V & $\mathrm{V}$ & 1 & $\mathrm{~V}$ & & & \\
\hline Crataegus laevigata. . & II & + & & II & & & \\
\hline Potentilla erecta .... & II & II & III & II & & & \\
\hline Calluna vulgaris. .... & IV & IV & 1 & IV & & & + \\
\hline Hedera helix . . . . . . & III & II & II & II & IV & III & \\
\hline Sorbus torminalis ... & $\mathrm{V}$ & II & & III & & IV & \\
\hline Leucobryum glaucum & & II & II & & & III & \\
\hline Ilex aquifolium. ..... & II & $\mathrm{I}$ & & II & + & II & \\
\hline
\end{tabular}


Tableau I (suite)

\begin{tabular}{|c|c|c|c|c|c|c|c|}
\hline & 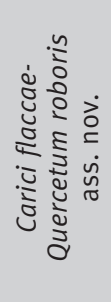 & 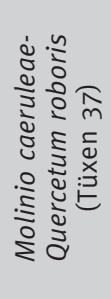 & 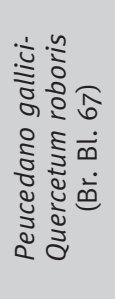 & 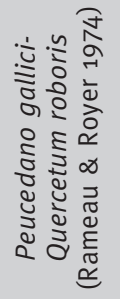 & 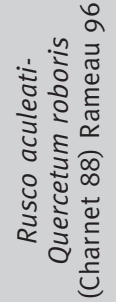 & 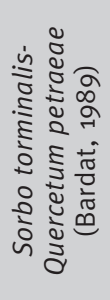 & 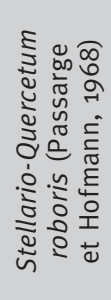 \\
\hline Hypericum pulchrum & III & I & II & IV & & + & \\
\hline $\begin{array}{l}\text { Thuidium } \\
\text { tamariscinum. . . . . . }\end{array}$ & IV & IV & II & & & III & \\
\hline Crataegus monogyna & IV & I & & II & 1 & II & \\
\hline Teucrium scorodonia & II & II & IV & IV & & V & \\
\hline Pleurozium schreberi & & III & II & & & II & \\
\hline Anemone nemorosa. . & III & + & & & IV & IV & IV \\
\hline Convallaria maialis. . . & II & + & & & + & III & III \\
\hline Hypnum cupressiforme & II & $\mathrm{V}$ & II & & & II & III \\
\hline $\begin{array}{l}\text { Polygonatum } \\
\text { multiflorum . . . . . . }\end{array}$ & III & & & & III & II & II \\
\hline Scleropodium purum & II & $\mathrm{V}$ & V & & + & III & $\mathrm{V}$ \\
\hline Polytrichum formosum & II & IV & & & + & IV & $\mathrm{V}$ \\
\hline Dicranum scoparium . & 1 & III & III & & & IV & III \\
\hline Eurhynchium striatum & IV & $\mathrm{I}$ & 1 & & & $\mathrm{I}$ & \\
\hline Melampyrum pratense & II & I & 1 & IV & & II & \\
\hline Holcus mollis. . . . . . . & 1 & + & IV & III & IV & II & IV \\
\hline Carex pilulifera ..... & 1 & III & III & II & & II & $\mathrm{V}$ \\
\hline Luzula pilosa. . . . . . . & & & & II & & & $\mathrm{V}$ \\
\hline Mespilus germanica. . & & & & II & & III & II \\
\hline Solidago virgaurea ... & 1 & & 1 & III & & $\mathrm{I}$ & \\
\hline Maianthemum bifolium & & & & & & + & $\mathrm{V}$ \\
\hline Milium effusum . . . . . & & & & & & & $\mathrm{V}$ \\
\hline Moehringia trinervia. . & & & & & & & $\mathrm{V}$ \\
\hline Mycelis muralis . . . . . & & & & & & & III \\
\hline Stellaria holostea. . . . & & & & & & & IV \\
\hline Rubus idaeus . . . . . & & & & & & & $\mathrm{V}$ \\
\hline
\end{tabular}

acidiphile correspondant typiquement au Molinio caeruleae-Quercetum roboris (Tüxen, 37) Scamoni et Passarge 59 et l'autre en milieu neutrocalcicole avec notre nouvelle association.

Mais on remarquera que notre nouvelle association comporte de nombreuses espèces des chênaies ligériennes (Alisier torminal, Peucédan de France entre autres), ce qui induit tout particulièrement sa proximité avec l'association du Peucedano gallici-Quercetum roboris $\mathrm{Br}-\mathrm{Bl}$ 67. De ce fait, nous proposons un rattachement du Carici flaccae-Quercetum roboris ass. nov. à l'alliance 
du Quercion robori-pyrenaicae (Br.-Bl., P. Silva, Rozeira et Fontes 56) Rivas Martinez ex Bardat et al. 2005. En notant toutefois que, même dans ce cas, la présence des espèces neutro-calcicoles comme le Brachypode penné et la Laîche glauque peut poser problème.

\section{UNE PROXIMITÉ AVEC LES PRAIRIES À MOLINIE (SYNDYNAMIQUE)}

De par la composition du cortège végétal et surtout la nature du matériau parental des sols, cette association se rapproche de l'alliance du Molinion caeruleae W. Koch 26 décrite en milieu de prairies oligotrophes à mésotrophes sur sol minéral à para-tourbeux. Cette alliance pourrait dériver de groupements forestiers par suite de déboisement important ou d'incendies (De Foucault, 1984). Le retour à la forêt est pratiquement impossible du fait de la modification des conditions édaphiques (accentuation des conditions asphyxiantes) et de la concurrence végétale induite, entre autres, par la Molinie. De Foucault (op. cité) souligne d'ailleurs le caractère à tendance "forestier" de certaines espèces des prairies à Molinie comme Succisa pratensis, Serratula tinctoria, Stachys officinalis, Calamagrostis epigejos, Carex pallescens.

Notre nouvelle association pourrait constituer un des groupements forestiers desquels dérive le Molinion prairial. D'autres rapprochements entre Molinion caeruleae W. Koch 26 et groupements forestiers sont également possibles. Mais jamais dans les études citées par de Foucault ne figure cette combinaison Molinie, Brachypode penné et Laîche glauque.

\section{CONCLUSIONS}

Pendant très longtemps on a rattaché toutes les stations à Molinie abondante (en nappe ou en touradons) à l'association du Molinio caeruleae-Quercetum roboris (Tüxen, 37) Scamoni et Passarge 59 sans se préoccuper des autres espèces présentes.

Or cette association est caractéristique des milieux acides fortement engorgés mais aucunement des milieux riches hydromorphes et encore moins des stations à régime hydrique très contrasté en particulier de celles développées sur des sols fortement argileux.

La création d'une nouvelle association végétale caractéristique de ces milieux particuliers est donc nécessaire pour rendre compte de la composition floristique originale de ce groupement. Cette association où l'on trouve la combinaison Molinie bleue - Brachypode penné - Laîche glauque a été nommée Carici flaccae-Quercetum roboris ass. nov. hoc. loco. Le relevé B71

TABLEAU II Relevé floristique B71 complet

(entre parenthèses les coefficients d'abondance-dominance selon Braun-Blanquet)

Strate arborescente : Quercus robur (3), Pyrus pyraster (1), Sorbus torminalis (3).

Strate arbustive : Sorbus torminalis (3), Quercus robur (3), Frangula alnus (2), Prunus spinosa (+), Juniperus communis (+), Crataegus laevigata (1), Malus sylvestris (1), Crataegus monogyna (1), Carpinus betulus (+), llex aquifolium (+).

Strate herbacée : Molinia caerulea (4), Brachypodium pinnatum (3), Carex flacca (2), Lonicera periclymenum (3), Rubus fruticosus (1), Stachys officinalis (1), Calluna vulgaris (1), Melampyrum pratense (1), Festuca heterophylla (1), Pulmonaria longifolia (+), Peucedanum gallicum (+), Rosa arvensis (+), Carex pilulifera (+), Erica scoparia (+), Hypericum pulchrum (+), Serratula tinctoria (+), Hedera helix (+), Poa trivialis (+).

Strate muscinale : Thuidium tamariscinum (1), Eurhynchium striatum (1), Polytrichum formosum (1), Scleropodium purum (1). 
(tableau II), réalisé dans le Bois la Biche à l’ouest de Châteauroux (Indre), est désigné comme holotypus de cette association.

En raison de la présence régulière d'espèces acidiphiles du Quercion, de l'engorgement prononcé de ses sols et du caractère ligérien du groupement végétal, cette association sera rattachée à l'alliance du Quercion robori-pyrenaicae (Br.-Bl., P. Silva, Rozeira et Fontes 56) Rivas Martinez ex Bardat et al. 2005.

Cette association décrite dans l'Orléanais et dans le Berry existe certainement dans d'autres régions dans des conditions édaphiques, topographiques et climatiques similaires. Leur recherche est donc à faire.

Alain BRÊTHES

Pôle Recherche d'Orléans OFFICE NATIONAL DES FORÊTS

100 boulevard de La Salle - BP 18

F-45760 BOIGNY-SUR-BIONNE

(alain.brethes@onf.fr)

\section{Remerciements}

L'auteur remercie vivement Messieurs R. Bœuf (ONF), C. Gauberville (IDF) et F. Olivereau (DREAL Centre) pour leur aide à la préparation de cette proposition.

\section{BIBLIOGRAPHIE}

BARDAT (J.), BIORET (F.), BOtINEAU (M.), BOULLET (V.), DELPECH (R.), GÉHU (J.-M.), HAURY (J.), LACOSTE (A.), RAMEAU (J.-C.), ROYER (J.-M.), ROUX (G.), TOUFFET (J.). - Prodrome des végétations de France. Paris : Muséum national d'histoire naturelle, 2004. - 171 p. (Patrimoines naturels, vol. 61).

BRAUN-BLANQUET (J.). - La Chênaie acidophile ibero-atlantique (Quercion occidentale) en Sologne. Communication $\mathrm{n}^{\circ}$ 178. - Montpellier : Station internationale de géobotanique méditerranéenne et alpine, 1967. - pp. 53-87.

BRÊTHES (A.). - Les stations forestières de la Champagne berrichonne et du Boischaut-sud (département du Cher et de l'Indre). - Document ONF, 2003. - 587 p.

BRÊTHES (A.). - Les types de station forestière de l'Orléanais. - Document ONF, 1993. - 400 p.

CHARNET (F.). - Catalogue des types de station forestière du Perche. - Publication du CRPF d'île-de-France et du Centre, 1988. - 583 p.

FOUCAULT (B. de). - Systémique, structuralisme et synsystématique des prairies hygrophiles des plaines atlantiques françaises. - Rouen : Université de Haute-Normandie, 1984. - 675 p. +246 tableaux (Thèse).

GAUBERVILLE (C.). - Les habitats forestiers en région Centre. Description, détermination et répartition provisoire. - Recherches naturalistes en Région Centre, $\mathrm{n}^{\circ} 12,2003$, pp. 3-49.

PASSARGE (H.), HOFMANN (G.). - Pflanzengesellschaften des nordostdeutschen Flachlandes II. Pflanzensoziologie, 16. - Jena : G. Fischer Verlag, 1968. - pp. 1-298.

RAMEAU (J.-C.). - Réflexions syntaxonomiques et synsystématiques au sein des complexes sylvatiques français. - Nancy : ENGREF, 1996. - $230 \mathrm{p}$.

RAMEAU (J.-C.), ROYER (J.-M.). - Les forêts acidiphiles du sud-est du Bassin parisien. In : "La végétation des forêts acidiphiles", Colloques phytosociologiques 3 (1974), 1975. - pp. 319-340 + 3 tableaux. 
SCAMONI (A.), PASSARGE (H.). - Gedanken zu einer natürlichen Ordnung der Waldgesellschaften. - Archiv für Forstwesen, vol. 8, 1959, pp. 386-426.

SOLOMESHCH (A.I.), GRIGORJEV (I.N.), KHAZIAKHMETOV (R.M.). - Syntaxonomy of South Urals forests. IV. Order Fagetalia sylvaticae. - M., Dep. in Viniti 12.10.89, N6234-B89, 1989. - 21 p.

TÜXEN (R.). - Die Pflanzengesellschaften Nordwestdeutschlands. - Mitteilungen der Floristisch-Soziologischen Arbeitsgemeinschaft in Niedersachsen, vol. 3, 1937, pp. 3-170.

\section{LE CARICI FLACCAE-OUERCETUM ROBORIS asS. nOV. UNE NOUUELLE RSSOCIATION PHYTOSOCIOLOGIOUE EN REGION CENTRE [Résumé]}

Une nouvelle association des chênaies humides a été décrite dans le Berry sous le nom de Carici flaccaeQuercetum roboris ass. nov. Elle correspond à la juxtaposition d'espèces hygro-tolérantes dont la Molinie bleue (Molinia caerulea (L.) Moench) et d'espèces considérées comme neutrocalcicoles dont le Brachypode penné (Brachypodium pinnatum (L.) Beauv.) et la Laîche glauque (Carex flacca Schreb.) en particulier. Sont présentés les caractères écologiques des milieux où cette association a été observée. Le rattachement à une alliance phytosociologique est discuté.

THE CARICI FLACCAE-OUERCETUM ROBORIS aSS. nOV. A NEW PHYTOSOCIOLOGICAL RSSOCIATION IN THE CENTRE REGION OF FRANCE [Abstract]

A new association of moist oak forests has been described in the Berry region in France under the name Carici flaccae-Quercetum roboris ass. nov. It is the juxtaposition of hygrotolerant species including purple moor-grass (Molinia caerulea (L.) Moench) and species considered to be neutral-calcicoles including in particular Tor grass (Brachypodium pinnatum (L.) Beauv.) and heath sedge (Carex flacca Schreb.). The ecological characteristics of the environments where this association is found are described. The author discusses ascribing this association to a phytosociological alliance. 


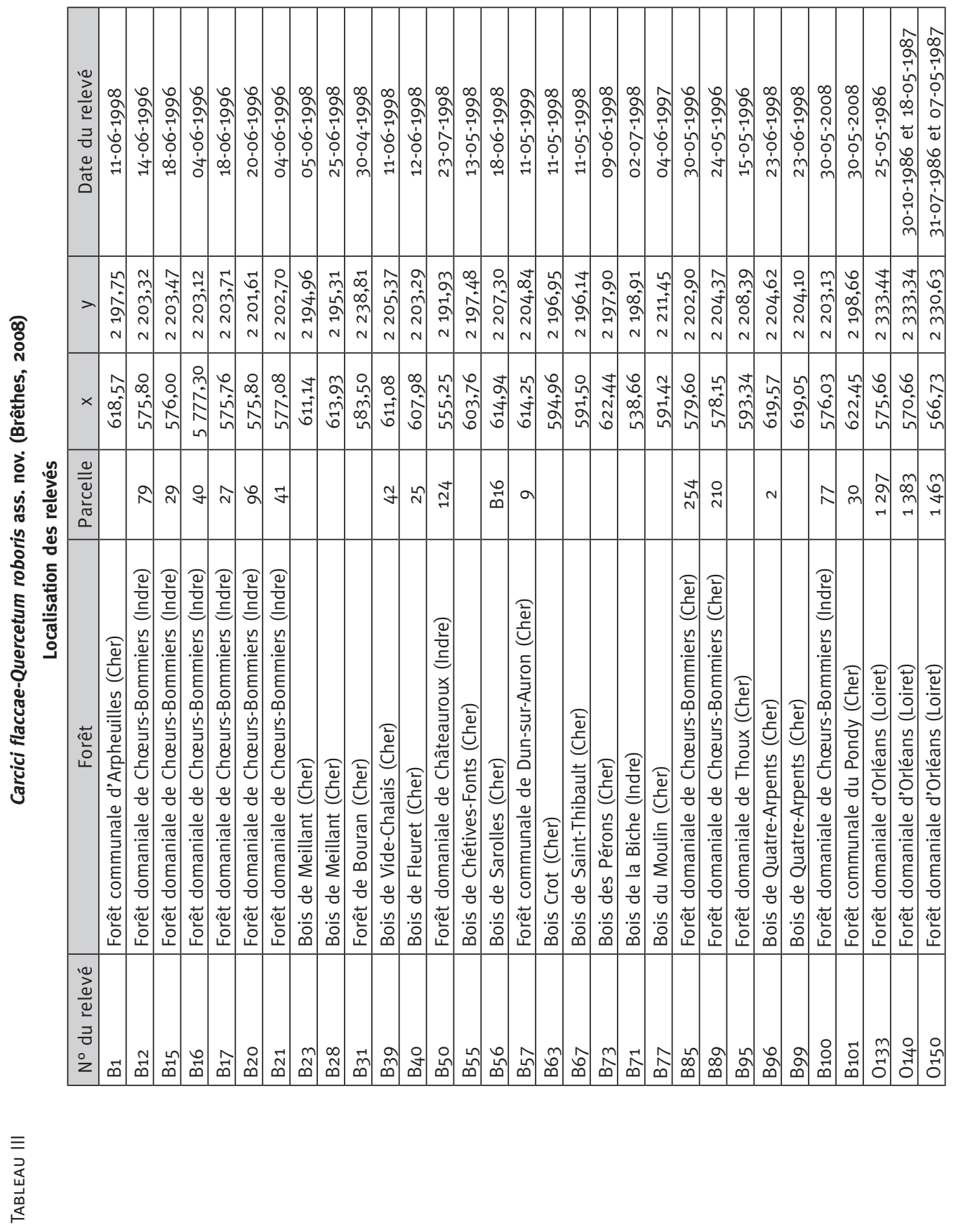


Session 1 - L'actualité de la phytosociologie et les découvertes

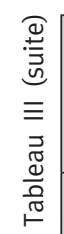

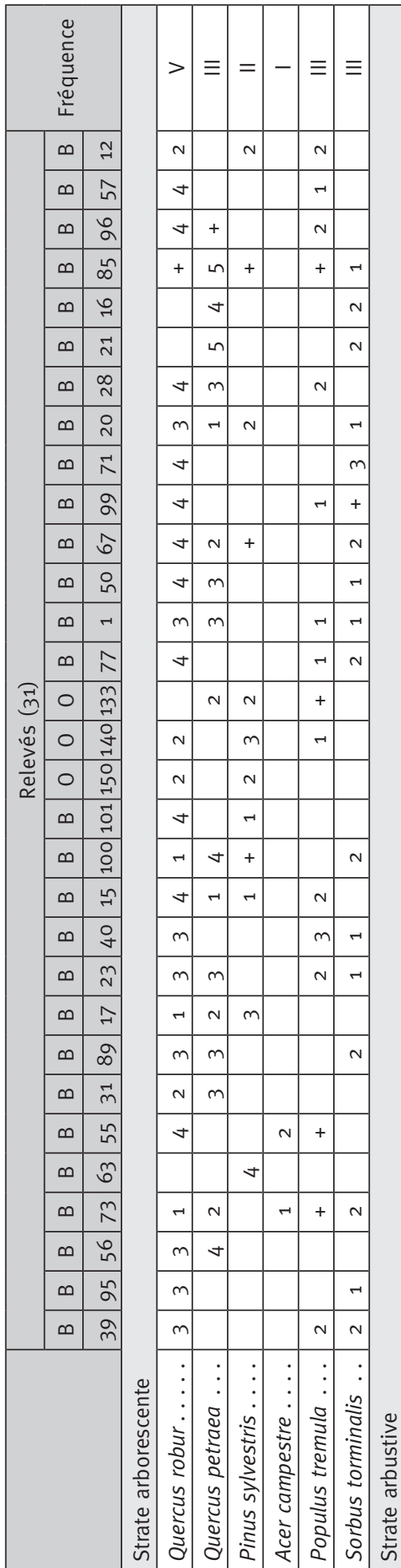

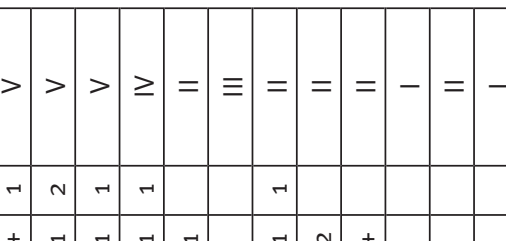

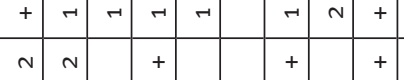

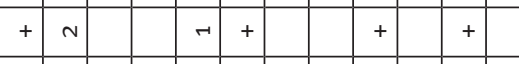

\begin{tabular}{l|l|l|l|l|l|l|l|l|l|l|}
+ & + & 4 & & & & & & & & + \\
\hline+ & & 4 & & & + & + & & & & + \\
\hline
\end{tabular}

\begin{tabular}{|l|l|l|l|l|l|l|l|l|l|l|l|}
\hline & 4 & & 4 & & & & & & & + & + \\
\hline
\end{tabular}

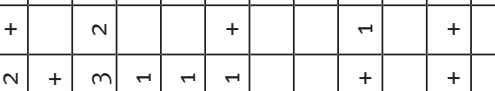

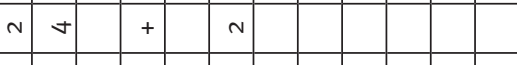

\begin{tabular}{cccc|c|c|c|c|c|c|c|c}
\hline & + & $m$ & - & & & & & & & & \\
\hline+ & & + & & & & & & & & + \\
\hline
\end{tabular}

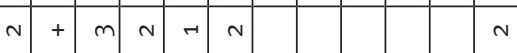

\begin{tabular}{lllllll}
4 & $r$ & $m$ & - & + \\
\hline
\end{tabular}

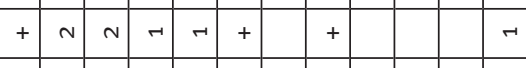

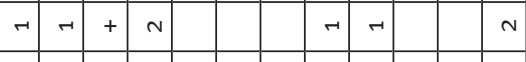

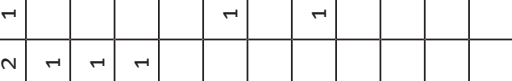

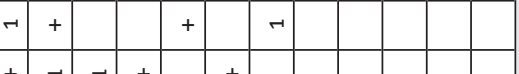

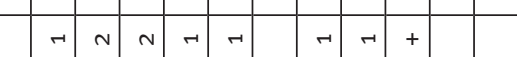

\begin{tabular}{|c|c|c|c|c|c|c|c|c|c|c|}
\hline$n$ & 4 & 4 & 4 & & & & & & & + \\
\hline$n$ & & $m$ & + & & + & & & & & \\
\hline
\end{tabular}

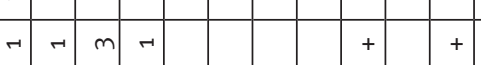

$n m-n+n$

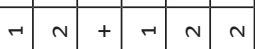

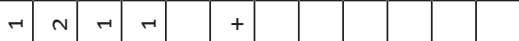

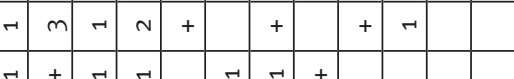

\begin{tabular}{ll|l|l|l|l|l|l|l} 
& $n$ & $m$ & $h$ & & $n$ & + & +
\end{tabular}

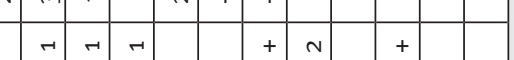

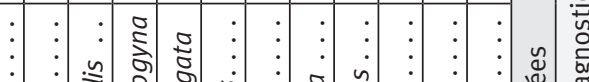

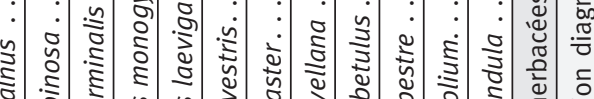

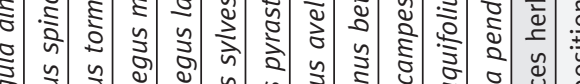

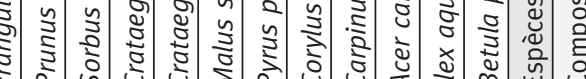

$>\quad>\geq$

\begin{tabular}{|l|l|l|l|}
\hline$n$ & $N$ & $N$ & + \\
\hline$m$ & $m$ & $N$ & - \\
\hline$m$ & $m$ & $N$ & $N$ \\
\hline
\end{tabular}

\begin{tabular}{|l|l|l|l|}
\hline$y$ & $n$ & + & + \\
\hline$m$ & $m$ & $n$ & $n$ \\
\hline
\end{tabular}

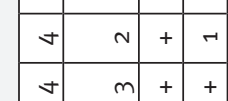

\begin{tabular}{|l|l|l|l|}
\hline & $m$ & + & + \\
\hline $\ln$ & $n$ & + & + \\
\hline & & & \\
\hline
\end{tabular}

\begin{tabular}{|ll|l|l|}
\hline$+v$ & $n$ & & \\
\hline+ & $n$ & + \\
\hline & $m$ & + & $n$ \\
\hline
\end{tabular}

\begin{tabular}{|l|l|l|l|}
\hline+ & $n$ & 4 & 4 \\
\hline+ & $m$ & + & $h$ \\
\hline$A$ & $n$
\end{tabular}

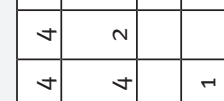

$m$ o $m$ n

\begin{tabular}{|l|l|l|l|}
\hline$\rightarrow$ & + & + & + \\
\hline$m$ & $m$ & & + \\
\hline
\end{tabular}

\begin{tabular}{|l|l|l|}
\hline$m$ & $m$ & + \\
\hline+ & $n$ & - \\
\hline
\end{tabular}

\begin{tabular}{|l|l|l|}
\hline $\ln$ & $\mathrm{n}$ & $\mathrm{N}$ \\
\hline
\end{tabular}

\begin{tabular}{|l|l|l|l|}
\hline$m$ & $m$ & + & \\
\hline$m$ & $m$ & $n$ & -1
\end{tabular}

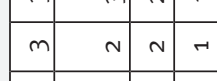

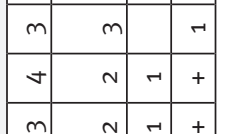

\begin{tabular}{|c|c|c|c|}
\hline$y$ & $n$ & 4 & + \\
\hline+ & $\checkmark$ & $n$ & \\
\hline+ & $m$ & $n$ & + \\
\hline
\end{tabular}

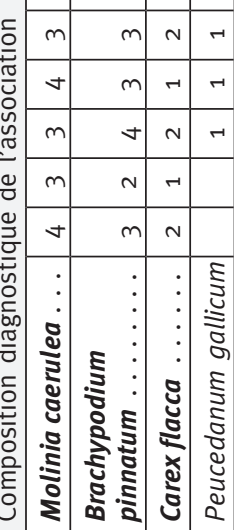


ALAIN BRÊTHES

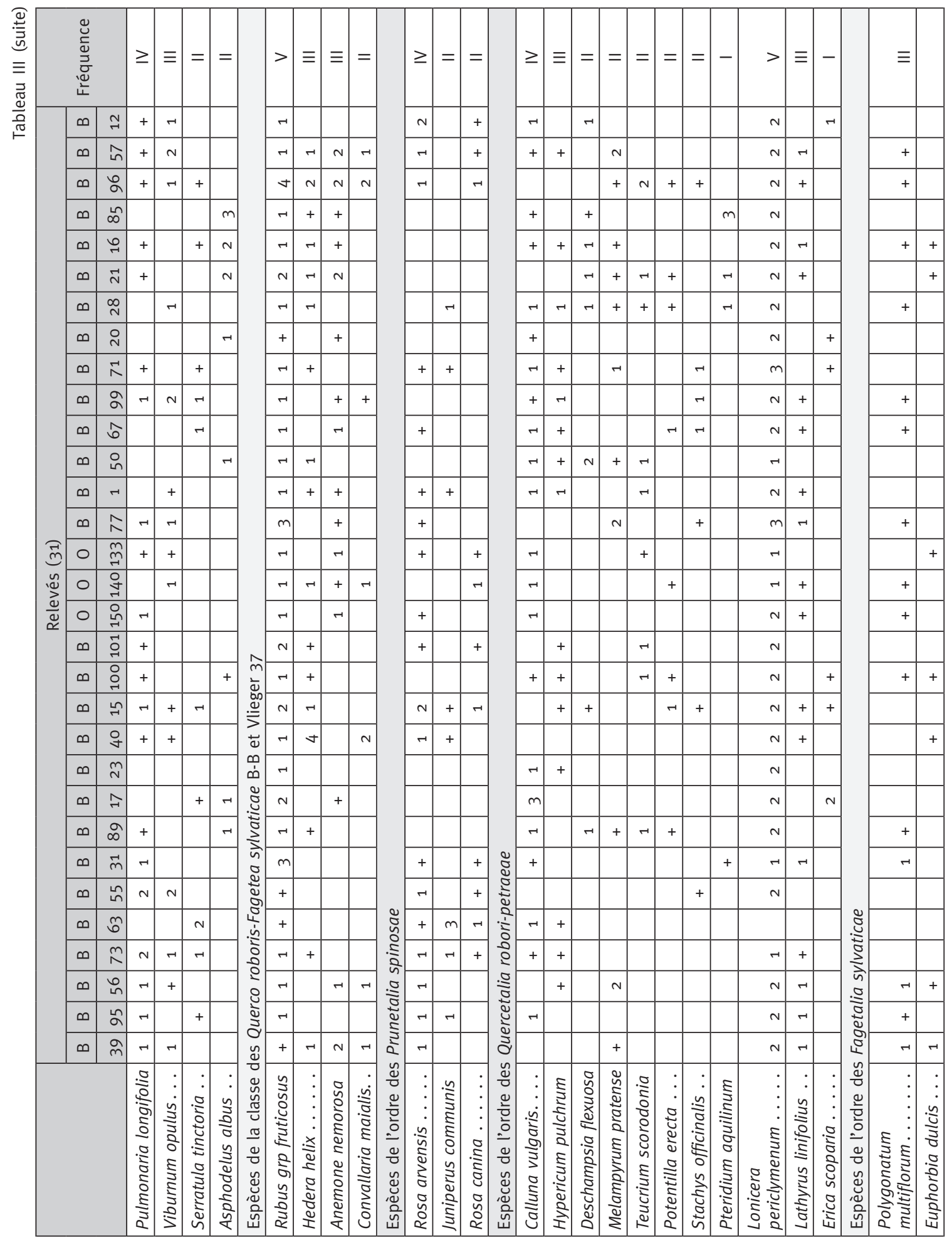


Session 1 - L'actualité de la phytosociologie et les découvertes

\begin{tabular}{|c|c|c|c|c|c|c|c|c|c|c|c|c|c|c|c|c|c|c|c|c|c|c|c|c|c|c|c|}
\hline & 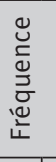 & & & -- & - & - & - & $\geq$ & - & - & $=$ & - & $\equiv$ & - & $=$ & $\geq$ & & & & & & & & & & & \\
\hline & & $\approx$ & & & & & & $r$ & & & & + & + & & $r$ & + & & & & & -1 & & & & & -1 & \\
\hline & $\infty$ & in & & & & + & & -1 & & & & & & & & $r$ & & & & & & & & & + & & + \\
\hline & $\infty$ & ฉ & & & + & & & + & + & & & & + & & & + & & & & & & & & & & + & \\
\hline & $\infty$ & $\stackrel{n}{\infty}$ & & & + & & & + & & & & & + & & & + & & + & & & & & & & & & \\
\hline & $\infty$ & 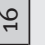 & & & + & + & & & & & + & + & $\rightarrow$ & & & + & & & -1 & & & & & & & & \\
\hline & $\infty$ & $\vec{N}$ & & & & & & $r$ & & & & & $r$ & & & & & & $r$ & & & & & & & & \\
\hline & $\infty$ & $\stackrel{\infty}{\sim}$ & & + & & & & -1 & & & & & $r$ & & $r$ & $N$ & & & $N$ & & & & & $r$ & & & \\
\hline & $\infty$ & ํ. & & & & & & -1 & & & & & $N$ & & $r$ & $r$ & & & & & & & & & & & \\
\hline & $\infty$ & $\vec{\lambda}$ & & & & & & -1 & & & & & $\rightarrow$ & & -7 & $r$ & & & & & & & & + & & & \\
\hline & $\infty$ & ลे & & & & & & & & & & & & & & + & & & & & & & & & & & \\
\hline & $\infty$ & $\hat{\sigma}$ & & & & + & & + & & & & & & & & & & & -1 & & & & & & & & \\
\hline & $\infty$ & in & & & & & & 7 & & & & & $N$ & & -1 & $r$ & & & & & & & & & & & \\
\hline & $\infty$ & $-T$ & & & & & & $\rightarrow$ & + & & + & & + & + & & $r$ & & + & & & & & & & & & \\
\hline & $\infty$ & 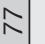 & & & & & & $r$ & & & & & & 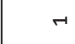 & & $r$ & & & & $r$ & & + & & & & + & \\
\hline $\overrightarrow{\tilde{\sigma}}$ & 0 & $\underset{\sim}{m}$ & & & & & & & & & $N$ & & & & $N$ & $r$ & & & & & & & & & & & \\
\hline$\stackrel{\mathscr{J}}{\supset}$ & 0 & $\begin{array}{l}\text { Oे } \\
\text { Y }\end{array}$ & & & & & + & & & & & & + & $r$ & $N$ & $N$ & & & & & & + & & & & & \\
\hline$\frac{\mathbb{E}}{\mathscr{\Phi}}$ & 0 & 욤 & & & & + & & $r$ & & & $r$ & & . & & -1 & $\rightarrow$ & & & & & & & & & & & \\
\hline & $\infty$ & 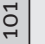 & & & & & & & & & & & + & & + & + & & & + & & & & & & & & + \\
\hline & $\infty$ & ঃ & & & & & & & & & & & & & & & & & & & & & & & & & \\
\hline & $\triangleright$ & $\stackrel{\sim}{\sim}$ & & & & & & -1 & & & & & & & + & + & & & & & & & & & & & \\
\hline & $\infty$ & 워 & & & & & & $\rightarrow$ & + & & & & & + & & $\pi$ & & & & & & & $N$ & & & + & \\
\hline & $\infty$ & $\stackrel{m}{\sim}$ & & + & -1 & & & + & & & . & & $r$ & & + & $\rightarrow$ & & & + & & & & & $r$ & & & \\
\hline & $\infty$ & $\hat{\exists}$ & & & & & & & & & $N$ & & & & $r$ & & & & & & & & & & & & \\
\hline & $\infty$ & ळ & & & & + & & -1 & + & & + & & + & & + & -1 & & & + & & + & & & + & & & \\
\hline & $\infty$ & $\vec{m}$ & & & & & & $\rightarrow$ & + & & & & & & & & & & & & & & & & & & \\
\hline & $\infty$ & in & & & & & & $N$ & + & & 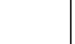 & & & + & & $N$ & & + & & & & + & & & & -1 & $r$ \\
\hline & $\infty$ & ๓ీ & & & & + & & & & $N$ & $N$ & & & + & $N$ & & & & & & & & & & & $N$ & \\
\hline & $\infty ?$ & $\stackrel{m}{\wedge}$ & & & & & & & & & & & & + & + & + & & & & $r$ & & & + & & & & \\
\hline & $\infty$ & in & & & & & & $\rightarrow$ & & & & & & & + & + & & & & & & & & & $r$ & & \\
\hline & $\infty$ & น & & & & & & -1 & & & & & & & & + & & & & $m$ & & & + & & & & \\
\hline & $\infty$ & mे & & & & & & $N$ & & & & & & & & $r$ & & & & + & & & -1 & & & & \\
\hline & & & 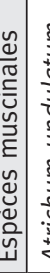 & 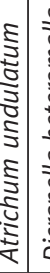 & 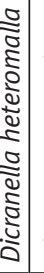 & 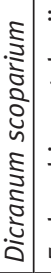 & 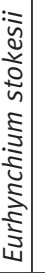 & 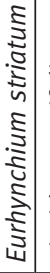 & 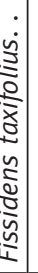 & 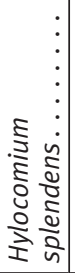 & 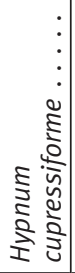 & 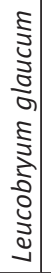 & 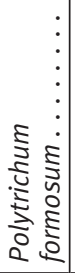 & 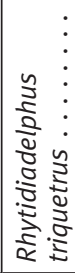 & 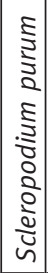 & 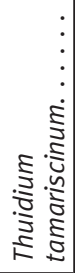 & 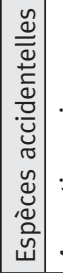 & 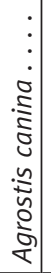 & 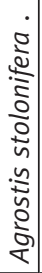 & 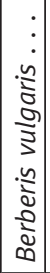 & 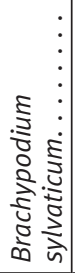 & 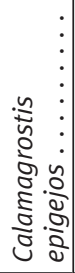 & 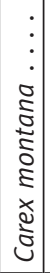 & 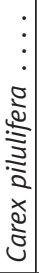 & 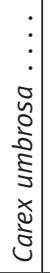 & 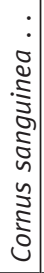 & 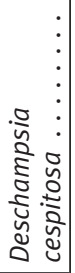 \\
\hline
\end{tabular}




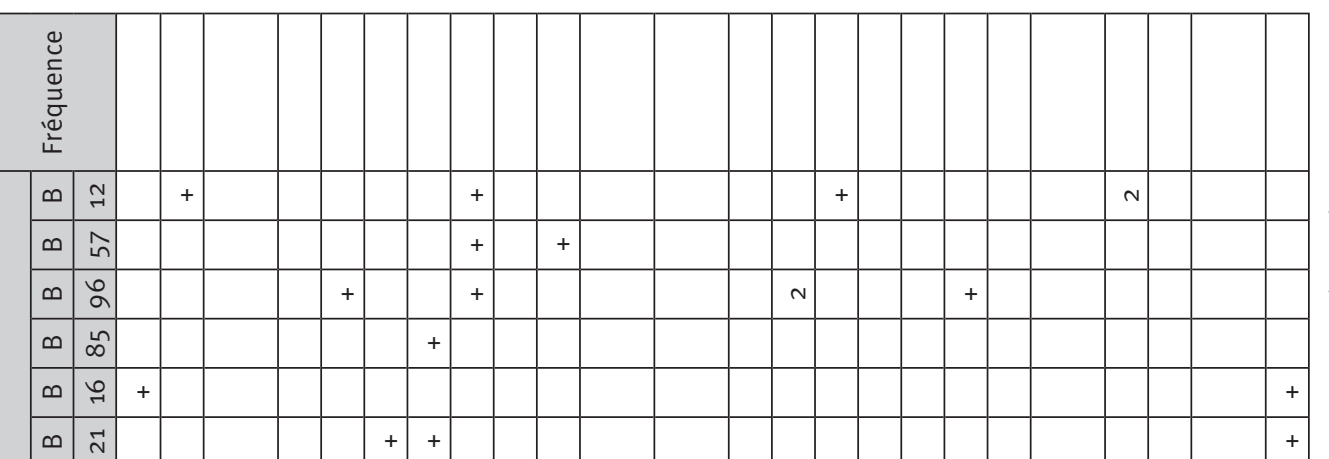

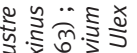
รำㅇำ

2⿺辶千

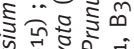

औิ

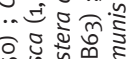

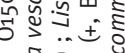

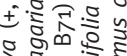

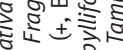

०

है ฉ है 응

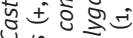

․ำ

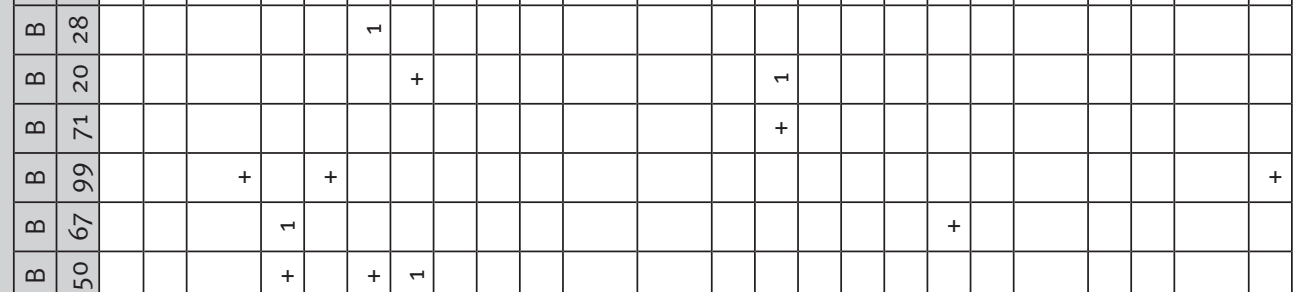

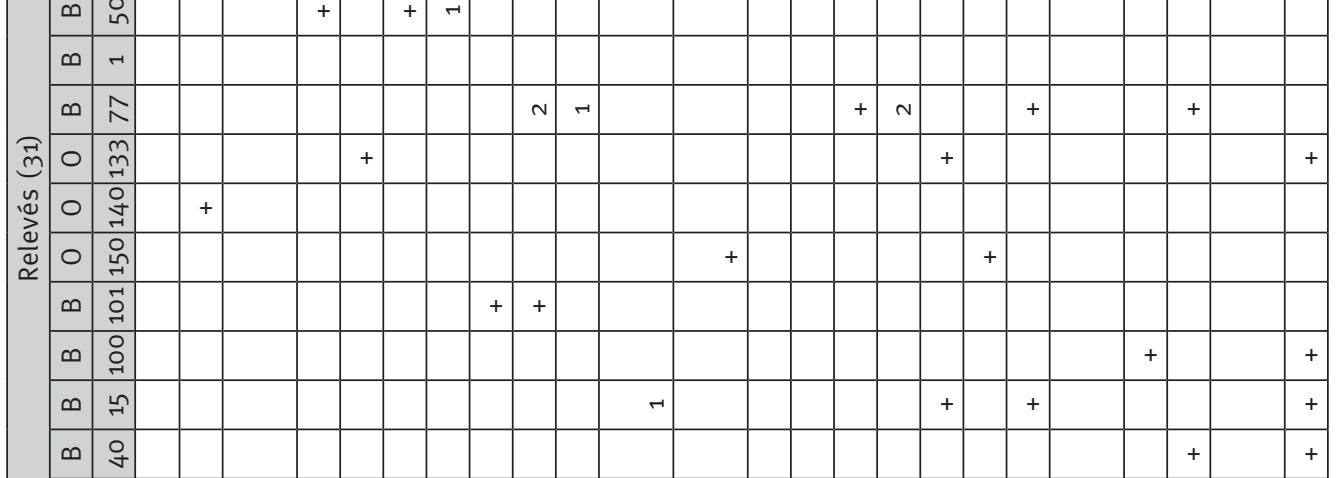

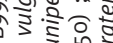

+ำำ

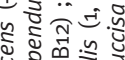

造这视

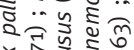

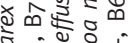

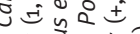

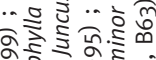

वें... हो

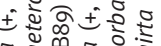

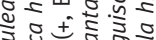

논

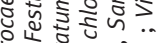

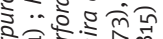

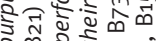

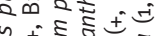

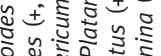

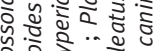

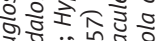

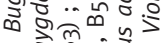

$\infty \stackrel{m}{\sim}$

$\infty$ సิ

$\infty$

合言品

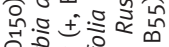

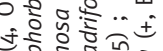

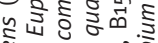

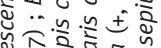

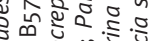

o

$\infty$ นก

$+++$

$\infty$ กิ

$+$

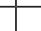

ง

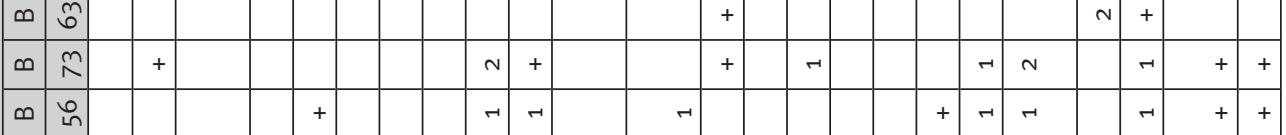

$\infty$ ผ

N $+t^{+}++$

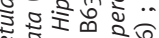

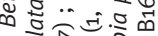

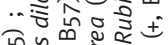

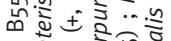

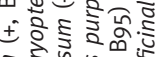

हो

$\infty$ ํ.

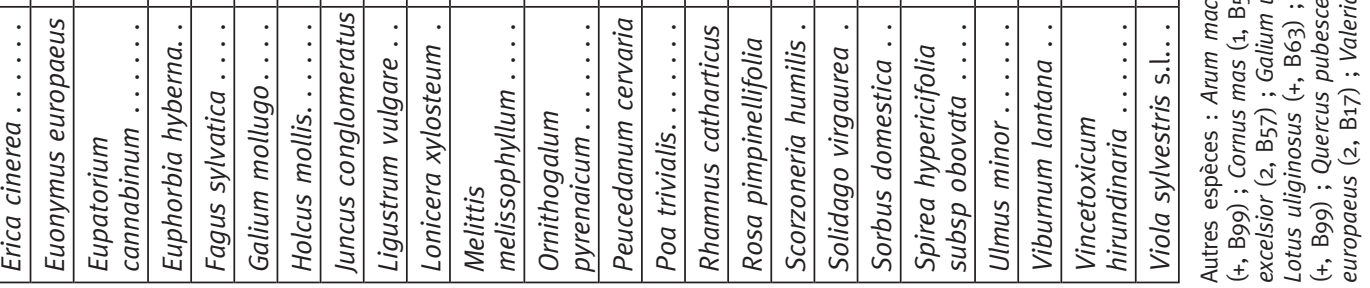

